\title{
Interaction Between Donor and Recipient Age in Determining the Risk of Chronic Renal Allograft Failure
}

\author{
Herwig-Ulf Meier-Kriesche, MD, ${ }^{*+}$ Diane M. Cibrik, MD, * Akinlolu O. Ojo, MD, * \\ Julie A. Hanson, MPH, "John C. Magee, MD, * Steven M. Rudich, MD, * \\ Allan B. Leichtman, $M D$, * and Bruce Kaplan, $M D^{*+}$
}

OBJECTIVES: Donor age is a known risk factor for chronic allograft failure (CAF) in renal transplant recipients. We have recently shown that advanced recipient age is also a risk factor for CAF. To investigate the interaction between donor and recipient age, we analyzed 40,289 primary solitary Caucasian adult renal transplants registered at the United States Renal Data System (USRDS) from 1988 to 1997.

DESIGN: CAF was defined as allograft loss beyond 6 months posttransplantation, censored for death, recurrent disease, acute rejection, thrombosis, noncompliance, infection, or technical problems. Cox proportional hazards models were used to investigate the risk of allograft loss secondary to CAF. All models were corrected for 15 covariates including donor and recipient demographics, ischemic time, and human leukocyte antigen match. Donor and recipient age were categorized, and relative risk for allograft loss of the interaction between the obtained categorical covariates was evaluated.

SETTING: Retrospective data analysis using the USRDS.

PARTICIPANTS: All primary Caucasian renal transplant recipients from 1988 to 1997.

RESULTS: Patients aged 55 and older who received donor kidneys had a $110 \%$ increased risk of CAF (relative risk $(\mathrm{RR})=2.1,95 \%$ confidence interval $(\mathrm{CI})=1.9-2.3$, $P<.001)$ and recipients aged 65 and older had a $90 \%$ increased risk for $\mathrm{CAF}(\mathrm{RR}=1.9,95 \% \mathrm{CI}=1.61-2.1, P<$ .001 ), compared with the youngest reference groups. In addition, there was an additive and, in the long term, synergistic interaction between donor and recipient age in determining allograft loss.

CONCLUSIONS: Donor and recipient age had an independent, equivalently detrimental effect on renal allograft

From the "Departments of Medicine and Surgery, The University of Michigan, Ann Arbor, Michigan; and 'Department of Internal Medicine, University of Florida, Gainesville, Florida.

Address correspondence to Bruce Kaplan, MD, University of Florida, College of Medicine, Department of Internal Medicine, Division of Nephrology, 1600 SW Archer Road, Gainesville, FL 32610. survival. An overall additive and, in the long term (beyond 36 months posttransplant), synergistic deleterious effect on renal allograft survival was observed for the interaction of donor and recipient age. J Am Geriatr Soc 50:14-17, 2002.

Key words: renal; transplant; age; nephropathy

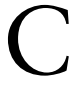
hronic renal allograft failure (CAF) is the major cause of late allograft failure after renal transplantation. ${ }^{1-4}$ Previous studies have demonstrated that donor age is a significant risk factor for chronic allograft failure. ${ }^{1,5,6}$ In addition, we have recently shown that, for Caucasian renal transplant patients, recipient age is an independent risk factor for CAF. ${ }^{7}$ A recent study by Basar et al. ${ }^{8}$ reported a significantly lower allograft survival at 1 and 5 years in older patients receiving kidneys from donors aged 60 and older compared with from those younger than 60 . Similarly, Smits et al. ${ }^{5}$ have reported a persistent risk of allograft loss for transplants from older donors into older recipients. The lack of a control group (younger recipients receiving organs from older donors) limits these studies. Therefore, these studies establish that older donor age is a risk factor for chronic allograft loss but have not ascertained whether a synergy between older donor and recipient age exists.

Donor and recipient age may act together to affect renal allograft survival. The impact of recipient age on $\mathrm{CAF}^{7}$ may act through different mechanisms of injury than does donor age. ${ }^{9}$ We sought to determine whether an interaction exists between these two variables.

\section{MATERIALS AND METHODS}

To investigate the nature of the interaction between donor and recipient age in determining long-term renal allograft survival, we analyzed data from 40,289 adult primary solitary Caucasian renal transplant patients registered in the U.S. Renal Data System (USRDS) database between 1988 and 1997. African-American recipients were excluded from the analysis because of the strong independent impact that 
African-American race has on the development of chronic allograft failure. ${ }^{7}$ The data for the analysis were provided by the U.S. Renal Transplant Scientific Registry and supplemented with end-stage renal disease data from the USRDS. Patients were followed from transplantation date until allograft loss, death, or the study end date of June 30, 1998. The primary study endpoint was CAF, defined as allograft loss 6 months posttransplantation, and censored for patient death or allograft loss secondary to acute rejection, allograft thrombosis, infection, surgical complications, or recurrent disease. This definition of CAF comes closer to the definition of grafts lost to chronic allograft nephropathy than to the traditionally used definition of death-censored allograft survival, in that all attributable causes of allograft loss not related to chronic allograft nephropathy are eliminated. ${ }^{7,10}$ Secondary study endpoints included overall allograft survival, death-censored allograft survival, and death with functioning allograft. Overall allograft survival included all grafts lost to any cause of allograft loss or patient death.

Death-censored allograft survival was defined as allograft survival in which only allograft losses not secondary to patient death were considered. In this analysis, when a patient died with a functioning allograft, the patient was excluded at time of death from the analysis without being counted as study event.

Death with a functioning allograft was defined as death occurring during the follow-up with a working transplant, excluding deaths occurring after resuming dialysis. For this definition, patients were censored upon resuming dialysis.

Cox proportional hazards models adjusted for potential confounding factors, including a categorical interaction term between donor age and recipient age, evaluated the effect of donor and recipient age on the primary and secondary study endpoints. In this analysis, donor and recipient age were categorized as shown in Figure 1. In additional Cox proportional hazards models, donor and recipient age were treated as continuous variables. In addition to donor and recipient age, all Cox models were corrected for the following potential confounding factors: donor and recipient race, gender, cytomegalovirus serology, immunoglobulin $\mathrm{G}(\operatorname{IgG})$ antibody status, primary cause of endstage renal disease, donor source (cadaveric vs living), cold and warm ischemia times, human leukocyte antigen (HLA) mismatch, presensitization, cyclosporine versus tacrolimus treatment, induction versus no induction treatment, mycophenolate mofetil versus azathioprine, acute rejection within the first 6 months posttransplantation, and delayed allograft function. Delayed allograft function was defined as a need for one or more dialysis treatments in the first week posttransplantation.

A probability of type 1 error $(\alpha)=0.05$ was considered to be the threshold of statistical significance. For multiple comparisons, the threshold of statistical significance was corrected by the Bonferroni adjustment. All statistical analysis was performed using SPSS software (Version 7.0 for Windows 95, SPSS, Inc., Chicago, IL).

\section{RESULTS}

Both donor and recipient age were significant independent risk factors for the development of chronic allograft failure. If treated as continuous variables, donor age con-

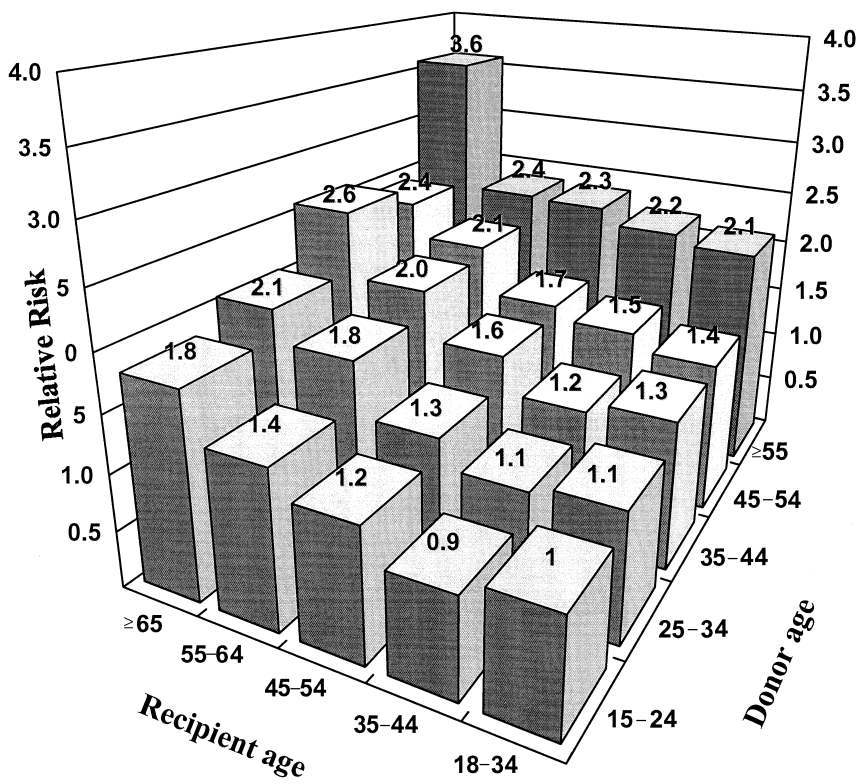

Figure 1. Relative risk for chronic allograft failure by donor and recipient age.

ferred a 1.018 relative risk (RR) per year $(95 \% \mathrm{CI}=$ $1.011-1.025, P<.001)$, and recipient age conferred a 1.014 RR per year $(95 \% \mathrm{CI}=1.008-1.020, P<.001)$ for the development of CAF (data not shown). When donor and recipient age were analyzed as categorical variables, the RR for the highest donor age group (55 and older) was $2.1(95 \% \mathrm{CI}=1.9-2.3, P<.001)$ for the development of CAF, whereas the RR for the highest recipient age group (65 and older) was 1.9 for the development of CAF $(95 \%$ CI $=1.6-2.1, P<.001$, Table 1$)$.

The RRs for the interaction between the categorized donor and recipient age variables are shown in Figure 1. The RR for CAF increased significantly and additively with increasing donor and recipient age.

Table 1. Relative Risk (RR) for Chronic Allograft Failure by Cox Proportional Hazard

\begin{tabular}{lccc}
\hline \multicolumn{1}{c}{ Variable } & RR & $95 \% \mathrm{Cl}$ & $P$-value \\
\hline Recipient age (18-35) & 1.0 & & Reference \\
36-45 & 1.0 & $0.9-1.1$ & ns \\
46-55 & 1.2 & $1.1-1.3$ & $<.001$ \\
56-65 & 1.4 & $1.3-1.5$ & $<.001$ \\
>65 & 1.9 & $1.6-2.1$ & $<.001$ \\
Donor age (15-25) & 1.0 & & Reference \\
26-35 & 1.2 & $1.1-1.3$ & $<.001$ \\
36-45 & 1.4 & $1.3-1.5$ & $<.001$ \\
$46-55$ & 1.5 & $1.4-1.6$ & $<.001$ \\
$>55$ & 2.1 & $1.9-2.3$ & $<.001$ \\
\hline
\end{tabular}

Note: Additional variables corrected for in the model but not displayed in the table are: donor and recipient gender, donor race, cold ischemia time, cause of end stage renal disease, human leukocyte antigen mismatch, immunosuppressive regimen, presensitization, cytomegalovirus serology, time on dialysis pretransplantation, year of transplant, and acute rejection.

$\mathrm{CI}=$ confidence interval; $\mathrm{ns}=$ nonsignificant 
Table 2. Relative Risk (RR) for Death with Functioning Graft by Cox Proportional Hazard

\begin{tabular}{lccc}
\hline \multicolumn{1}{c}{ Variable } & $\mathrm{RR}$ & $95 \% \mathrm{Cl}$ & $P$-value \\
\hline Recipient age (18-35) & 1.0 & & Reference \\
36-45 & 1.4 & $1.3-1.6$ & $\mathrm{~ns}$ \\
46-55 & 2.1 & $1.9-2.4$ & $<.001$ \\
56-65 & 3.4 & $3.1-3.8$ & $<.001$ \\
>65 & 5.4 & $4.7-6.2$ & $<.001$ \\
Donor age (15-25) & 1.0 & & Reference \\
26-35 & 1.0 & $0.9-1.1$ & $<.001$ \\
36-45 & 1.2 & $1.1-1.3$ & $<.001$ \\
$46-55$ & 1.3 & $1.1-1.4$ & $<.001$ \\
$>55$ & 1.4 & $1.2-1.5$ & $<.001$ \\
\hline
\end{tabular}

Note: Additional variables corrected for in the model but not displayed in the table are: donor and recipient gender, donor race, cold ischemia time, cause of end stage renal disease, human leukocyte antigen mismatch, immunosuppressive regimen, presensitization, cytomegalovirus serology, time on dialysis pretransplantation, and year of transplant.

$\mathrm{CI}=$ confidence interval; $\mathrm{ns}=$ nonsignificant.

As shown in Table 2, the RR for patient death with functioning allograft increased as expected to a high of 5.4 for the recipients aged 65 and older. In addition, donor age was a significant risk factor for patient death with functioning allograft. The RR for patient death for a donor age of 55 or older was 1.4.

The interaction between the categorized donor and recipient age variables from the Cox model for allograft survival beyond 36 months is shown in Figure 2. The interaction between donor and recipient age conferred a significant risk for long-term allograft loss. This interaction between donor and recipient age for long-term allograft survival was statistically significant $(P<.05)$ when donor and recipient age and the interaction term were entered as continuous variables into the Cox model.

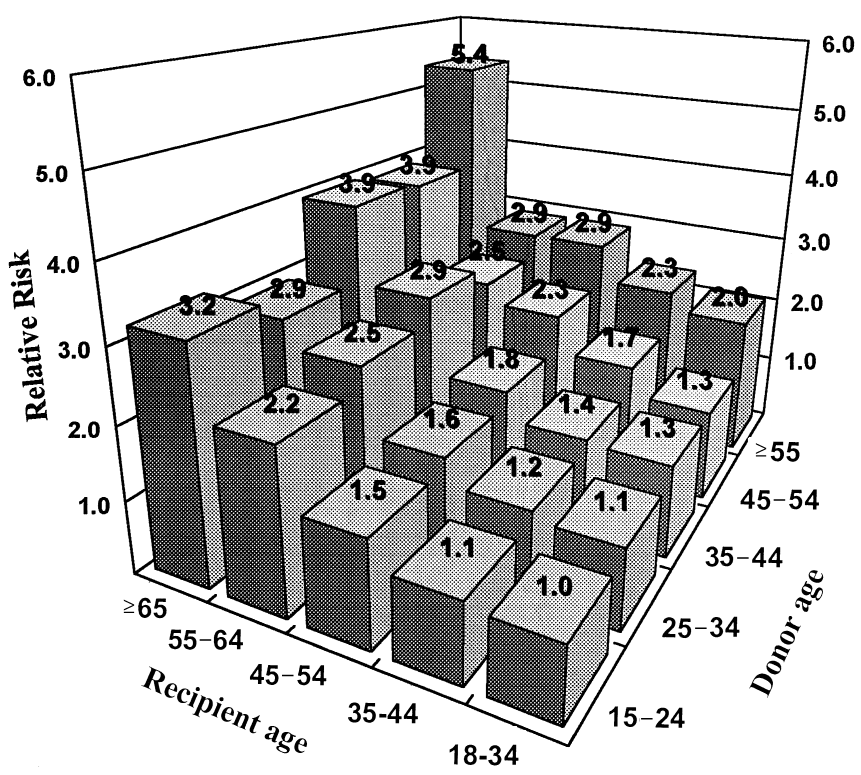

Figure 2. Relative risk for graft failure beyond 36 months by donor and recipient age.

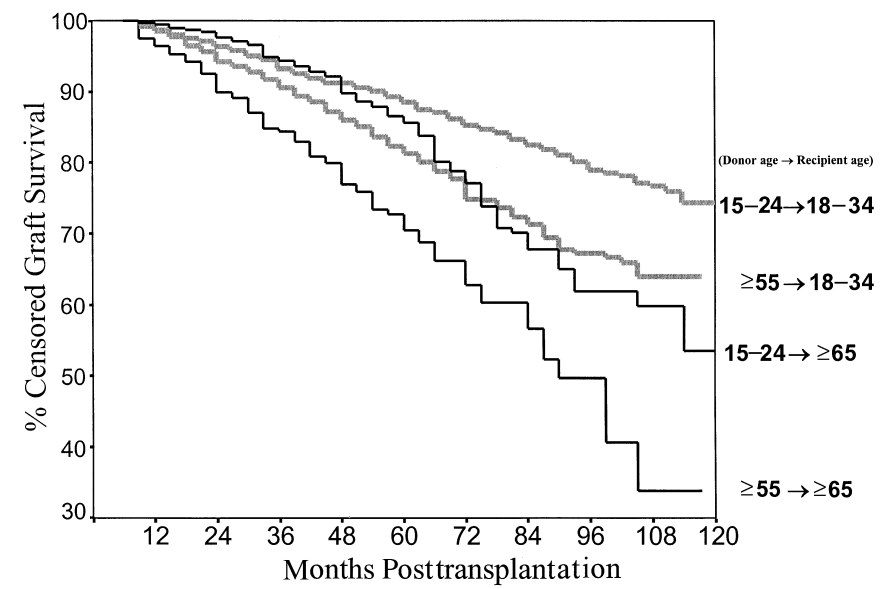

Figure 3. Death-censored graft survival beyond 6 months posttransplantation.

Figure 3 shows the Kaplan-Meier analysis of deathcensored allograft survival beyond 6 months posttransplantation with extreme donor and recipient age groups. A young donor kidney (aged 15-25) transplanted into a young recipient (aged 18-35) had the best long-term allograft survival. A young donor kidney (aged 15-25) transplanted into an older recipient (aged 65 and older) had allograft survival as good as that of a young recipient during the first 5 years posttransplantation, but after 5 years the allograft survival became significantly worse. An older donor kidney (aged 55 and older) transplanted into a young recipient (aged 18-35) had significantly worse allograft survival compared with a young kidney transplanted into the same age group, emphasizing the effect of donor age. Overall, an older donor kidney transplanted into an older recipient had by far the worst allograft survival.

\section{DISCUSSION}

Previous studies have reported on the detrimental effect of increased donor age on long-term renal allograft survival. $^{1-3}$ Recently, we demonstrated that recipient age is also a significant and independent risk factor for the development of CAF. ${ }^{7}$ It has also been noted that the exclusive allocation of kidneys from older donors to older recipients produces surprisingly poor results. ${ }^{8}$ In our present analysis, the detrimental effect of recipient age is of similar magnitude to that of donor age. Furthermore, donor and recipient age seem to have an additive effect for increasing the risk of chronic allograft failure, and, for allografts surviving beyond 3 years, the interaction becomes synergistic.

To date, our study is the first to quantify this potential interaction. The increasing age of the end-stage renal disease population and the consequently progressively increasing age of renal transplant recipients, combined with the growing use of older and more-marginal kidney donors, ${ }^{4}$ makes this a particularly important finding.

We would like to make clear that our analysis is not intended to address issues of allocation of donor kidneys but rather to describe and quantify the interaction of donor and recipient age in determining long-term renal allograft survival. Potential conclusions about allocation would 
have to include an evaluation of all other significant risk factors for allograft and patient survival, in addition to ethical and economical considerations.

The univariate Kaplan-Meier analysis of death-censored allograft survival beyond 6 months posttransplantation further strengthens our hypothesis of the interaction between donor and recipient age in causing long-term allograft failure. In fact, in the Kaplan-Meier analysis, for older patients receiving a young donor organ there is an accelerated allograft loss late after transplantation, especially after 5 years posttransplantation. From this analysis, in which acute rejection was not corrected for, on close observation there was a tendency toward better death-censored allograft survival from early after transplantation up until 4 years in older people receiving an ideal young kidney. This is probably an effect of the lower incidence of acute rejection in older people. In light of this, the late drop in death-censored allograft survival is even more impressive and points toward a process that is deleterious in the long term despite initially very favorable conditions. This observation strengthens our previously published hypothesis that the pathophysiological milieu of the aging recipient might have a deleterious long-term effect on transplanted kidneys. ${ }^{7}$ From the Kaplan-Meier analysis in Figure 3 , but also from the multivariate analysis, it is clear that older recipients receiving an older kidney experience a dramatic decline in allograft survival from early after transplantation. These observations suggest that the pathophysiological milieu of the aging body might exacerbate the intrinsic vulnerability of an older donor kidney and, subsequently, with time might also be detrimental to a younger donor kidney.

Although the multivariate analyses confirm that the observed interactions between donor and recipient age are independent of potential confounding variables, the univariate Kaplan-Meier analysis of donor-recipient pairings at the extremes of age is helpful in defining the timing of allograft loss and in graphically depicting and understanding the interaction.

The cause of the interaction between donor and recipient age in CAF has yet to be determined. Preexisting vasculopathy in an older donor kidney $y^{3,6,11}$ in combination with the older recipient's aging milieu may hasten the progression of chronic allograft nephropathy. Apolipoproteins, homocysteine, sex hormones, and T-cell derived growth factor- $\beta^{12-18}$ are a few mediators that undergo significant changes with aging and that might promote chronic allograft nephropathy and might constitute the "aging milieu."

Interestingly, donor age and recipient age are significant risk factors for patient death with a functioning allograft. It is not surprising that recipient age is associated with increase mortality posttransplantation. However, it is not intuitive why donor age affects patient survival. It is possible that worse clearance translates into more hypertension or is intrinsically a risk factor for patient survival, but, based on our data, we are hesitant to speculate about the pathogenesis of this finding.

In summary, the risk of chronic allograft nephropathy dramatically increases via the interaction between donor and recipient age. Our observations strengthen the hypothesis that the aging milieu exacerbates the intrinsic vulnerability of an older donor kidney. The exact pathogenesis of the interaction between donor and recipient age and the possible impact on organ allocation will require further study.

\section{REFERENCES}

1. Gjertson DW. A multi-factor analysis of kidney graft outcomes at one and five years posttransplantation: 1996 UNOS Update. Clin Transpl 1996; 343-360.

2. Matas AJ, Burke JFJ, DeVault GAJ et al. Chronic rejection. J Am Soc Nephrol 1994;4:S23-S29.

3. Paul LC. Chronic allograft nephropathy: An update. Kidney Int 1999;56: 783-793.

4. U.S. Renal Data System. USRDS 1999 Annual Data Report. Bethesda, MD: The National Institute of Health, National Institute of Diabetes Mellitus and Digestive and Kidney Diseases, 1999.

5. Smits JM, De Meester J, Persijn GG et al. Long-term results of solid organ transplantation. Report from the Eurotransplant International Foundation. Clin Transpl 1996;109-127.

6. Halloran PF, Melk A, Barth C. Rethinking chronic allograft nephropathy: The concept of accelerated senescence. J Am Soc Nephrol 1999;10:167-181.

7. Meier-Kriesche HU, Ojo AO, Cibrik DM et al. Relationship of recipient age and development of chronic allograft failure. Transplantation 2000;70: 306-310.

8. Basar H, Soran A, Shapiro R et al. Renal transplantation in recipients over the age of 60: The impact of donor age. Transplantation 1999;67:11911193.

9. Halloran PF, Melk A, Barth C. Rethinking chronic allograft nephropathy: The concept of accelerated senescence. J Am Soc Nephrol 1999;10:167-181.

10. Ojo AO, Meier-Kriesche HU, Hanson JA et al. Mycophenolate mofetil reduces late renal allograft loss independent of acute rejection. Transplantation 2000;69:2405-2409.

11. Nakatsuji S, Yamate J, Sakuma S. Macrophages, myofibroblasts, and extracellular matrix accumulation in interstitial fibrosis of chronic progressive nephropathy in aged rats. Vet Pathol 1998;35:352-360.

12. Nakamura T, Fox-Robichaud A, Kikkawa R et al. Transcription factors and age-related decline in apolipoprotein A-I expression. J Lipid Res 1999;40: 1709-1718

13. Muller V, Szabo A, Viklicky O et al. Sex hormones and gender-related differences: Their influence on chronic renal allograft rejection. Kidney Int 1999; 55:2011-2020.

14. Rea IM, McNerlan SE, Alexander HD. CD69, CD25, and HLA-DR activation antigen expression on CD3+ lymphocytes and relationship to serum TNF-alpha, IFN-gamma, and sIL-2R levels in aging. Exp Gerontol 1999;34: 79-93.

15. Straub RH, Konecna L, Hrach $\mathrm{S}$ et al. Serum dehydroepiandrosterone (DHEA) and DHEA sulfate are negatively correlated with serum interleukin6 (IL-6), and DHEA inhibits IL-6 secretion from mononuclear cells in man in vitro: Possible link between endocrinosenescence and immunosenescence. J Clin Endocrinol Metab 1998;83:2012-2017.

16. Chiang AN, Chang CP, Chou YC et al. Differential distribution of apolipoprotein $\mathrm{E}$ in young and aged spontaneously hypertensive and stroke-prone rats. J Hypertens 1999;17:793-800.

17. Herrmann W, Quast S, Ullrich M et al. Hyperhomocysteinemia in high-aged subjects: Relation of B-vitamins, folic acid, renal function and the methylenetetrahydrofolate reductase mutation. Atherosclerosis 1999;144:91-101.

18. Ruiz-Torres MP, Bosch RJ, O'Valle F et al. Age-related increase in expression of TGF-beta1 in the rat kidney: Relationship to morphologic changes. J Am Soc Nephrol 1998;9:782-791. 